\title{
Correction to: A Review of Complications Due to the Use of Botulinum Toxin A for Cosmetic Indications
}

\author{
Nitin Sethi ${ }^{1}$ (D) Sukhbir Singh ${ }^{2} \cdot$ Koenraad DeBoulle $^{3} \cdot$ Eqram Rahman $^{4}$
}

Published online: 29 November 2021

(C) Springer Science+Business Media, LLC, part of Springer Nature and International Society of Aesthetic Plastic Surgery 2021

\section{Correction to: Aesth Plast Surg (2021) 45:1210-1220 https://doi.org/10.1007/s00266-020- 01983-w}

The authors would like to correct figure legends in this article. The figure legends should read.

Fig. 1 Typical "Mephisto" appearance on the right side after injection in the glabella and central frontal area, view at max lifting of brows-indication of point for injection of a correction dose of BoNTA (indicated by pencil point) at max frown after correction of Mephisto appearance. (Image courtesy Dr. K De Boulle)

Fig. 2 Typical spread of BoNTA into the left depressor labii inferioris muscle, with consequent inability to lower

The original article can be found online at https://doi.org/10.1007/ s00266-020-01983-w.

Nitin Sethi

drnitinsethi@gmail.com

Sukhbir Singh

Sukhi_1@yahoo.com

Koenraad DeBoulle

koen@doctordeboulle.be

Eqram Rahman

eqram.rahman@gmail.com

1 Present Address: Plastic and Cosmetic Surgery, Fortis Hospital Ludhiana, Punjab 141001, India

2 Resplendent the Cosmetic Studio, R-9, GK-1, New Delhi 110048 , India

3 Aalst Dermatology Clinic, Aalst, Belgium

4 Department of Plastic and Reconstructive Surgery, University College London and Royal Fre Hospital, Hampstead, London NW3 2QG, UK the left side of the lip creating an asymmetric smile. (Image courtesy Dr. K. De Boulle)

Fig. 3 Upon injection of crow's feet spread of BoNTA into the lifting muscles of corner of the mouth, resulting in aberrant smile, drooping of the corner of the mouth, and accentuation of the marionette line. (Image courtesy Ada Trindade de Almeida São Paulo, Brazil)

Fig. 4 Injection into the masseter muscle to reduce hypertrophy resulting in abscess formation at the injection sites. The abscesses were resistant to routine antibiotic treatment and needed drainage and antitubercular treatment to resolve the problem. (Image courtesy Dr. Ruchi Mutneja and Dr. Nitin Sethi)

In the results section, the sentence "A PRISMA Flow chart for the selection of articles is given in Fig. 1" should have referred to Fig. 5.

Publisher's Note Springer Nature remains neutral with regard to jurisdictional claims in published maps and institutional affiliations. 